\title{
Optically Coupled Mode-Locked Laser Array for Spectroscopy in InP Generic Integration
}

\author{
Mu-Chieh Lo* \\ University College London \\ London, UK, and \\ Universidad Carlos III de Madrid \\ Leganés, Spain \\ *mlo@ing.uc3m.es
}

\author{
Christoph Weber, Dominik Auth, \\ Patrick Fiala, and Stefan Breuer* \\ Institute of Applied Physics, \\ Technische Universität Darmstadt \\ Darmstadt, Germany \\ *stefan.breuer@physik.tu-darmstadt.de
}

\author{
Guillermo Carpintero* \\ Department of Electronic Technology, \\ Universidad Carlos III de Madrid \\ Leganés, Spain \\ *guiller@ing.uc3m.es
}

\begin{abstract}
We propose a two-element mode-locked laser array operating at $1.56 \mu \mathrm{m}$. Optical pulses with 14.5-ps width, spectral bandwidth exceeding $7 \mathrm{~nm}$, and RF line width of 260 $\mathrm{kHz}$ at $12.448 \mathrm{GHz}$ are experimentally reported.
\end{abstract}

Keywords-Diode laser arrays, Mode-locked lasers, Photonic integrated circuits

\section{INTRODUCTION}

Dual-comb spectroscopy (DCS) allows for high-speed sweeping of spectral fingerprints, which requires two optical frequency comb generators (OFCG) with slightly different comb separation frequencies and comb lines spectrally correlated to those of each other [1]. Such mode-locked OFCGs with intra-cavity phase shifters can offer further reliability, tunability and accessibility on a photonic integrated circuit (PIC) [2]. In this paper, we demonstrate two $12.5-\mathrm{GHz}$ repetition rate mode-locked lasers (MLL) in 6.5-mm ring cavities which are optically coupled on a single chip using an active-passive generic foundry approach [3]. Laser emission characterization results including optical spectra, radiofrequency $(\mathrm{RF})$ spectra and autocorrelation (AC) traces are presented.

\section{DEVICE DESCRIPTION}

The layout and photo of the proposed two-MLL array PIC are shown in Fig. 1(a) and Fig. 1(b), respectively. Each symmetric ring cavity is $6.5-\mathrm{mm}$ long corresponding to a repetition rate of $12.5 \mathrm{GHz}$, and is composed of one $30-\mu \mathrm{m}$ saturable absorber (SA), two 400- $\mu \mathrm{m}$ amplifier sections (SOA, semiconductor optical amplifier), 4000- $\mu$ m electrooptic phase modulator (EOPM) in four sections and other passive components. Between these active sections electrical isolation sections are inserted to avoid unwanted current flows. The deeply etched passive s-shaped and arc-shaped waveguides with $100-\mu \mathrm{m}$ bending radii form the ring geometry. One $3-\mathrm{dB} 2 \times 2$ MMI coupler guides the optical signals towards the cleaved facet. Out of the cavities, one MMI coupler combines the optical signal from each MLL followed by two output waveguides with/without booster amplifier SOA, respectively.

\section{RESULTS AND DISCUSSION}

The lower MLL (Ring1) is characterized by forward biasing its two SOA sections with a gain current ( $\mathrm{I}_{\mathrm{SOA}}$ ) and biasing the $\mathrm{SA}$ with a reverse voltage $\left(\mathrm{V}_{\mathrm{SA}}\right)$. The signal from the waveguide output (OUT) without booster is collected with a lensed fiber and sent to optical/electrical spectrum analyzers and autocorrelator. Fig. 1(c) shows the optical spectrum for $\mathrm{I}_{\mathrm{SOA}}=110 \mathrm{~mA}$ and $\mathrm{V}_{\mathrm{SA}}=1.9 \mathrm{~V}$, with a $-3-\mathrm{dB}$ comb width exceeding $7 \mathrm{~nm}$ despite the $>5-\mathrm{dB}$ dip at $1560 \mathrm{~nm}$. Fig. 1(d) shows the AC trace for the same operation, the deconvoluted pulse width amounts to 14.5 ps assuming a Gaussian shape.

The -3-dB spectral comb width is depicted in dependence on $\mathrm{I}_{\mathrm{SOA}}=60-180 \mathrm{~mA}$ and $\mathrm{V}_{\mathrm{SA}}=1.5-2.6 \mathrm{~V}$ in Fig. 2(a). Within $\mathrm{V}_{\mathrm{SA}}=1.6-2.4 \mathrm{~V}$ and $\mathrm{I}_{\mathrm{SOA}}=70-130 \mathrm{~mA}$, multimode lasing spectra are found whereby the spectral bandwidth can (a)
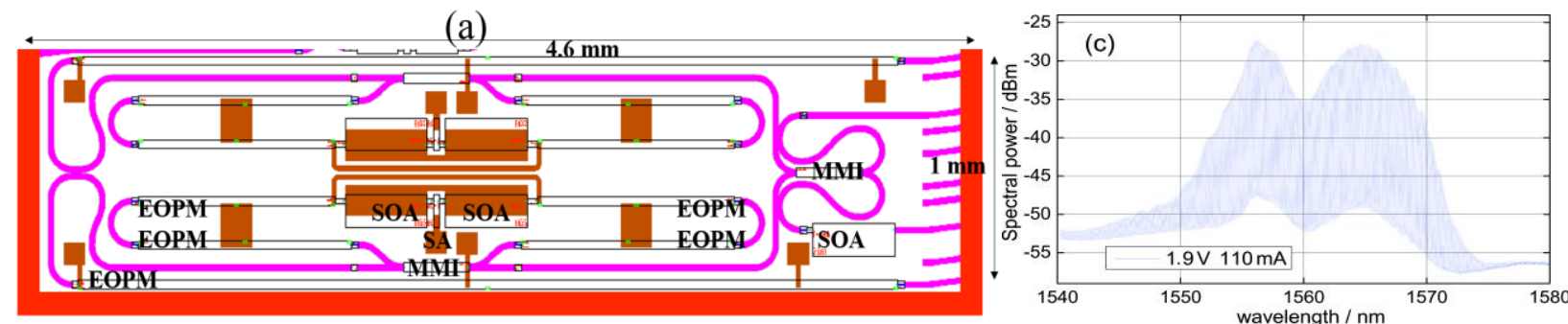

(b)
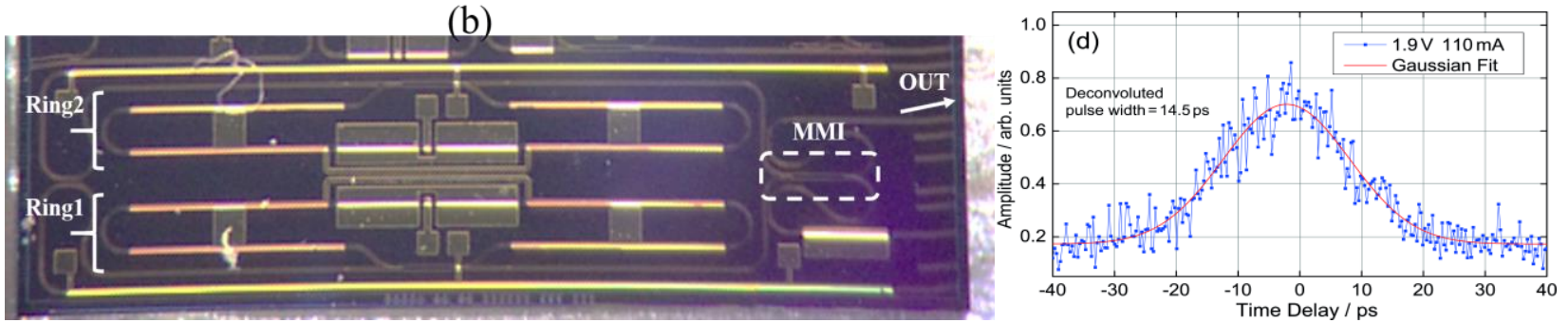

Fig. 1: (a) Layout of the dual-MLL PIC. (b) Photo of the dual-MLL PIC. (c) Measured optical spectrum, and (d) Measured $\mathrm{AC}$ trace for $\mathrm{I}_{\mathrm{SOA}}=110 \mathrm{~mA}, \mathrm{~V}_{\mathrm{SA}}=1.9 \mathrm{~V}$. operating at $1.5 \mu \mathrm{m}$. 

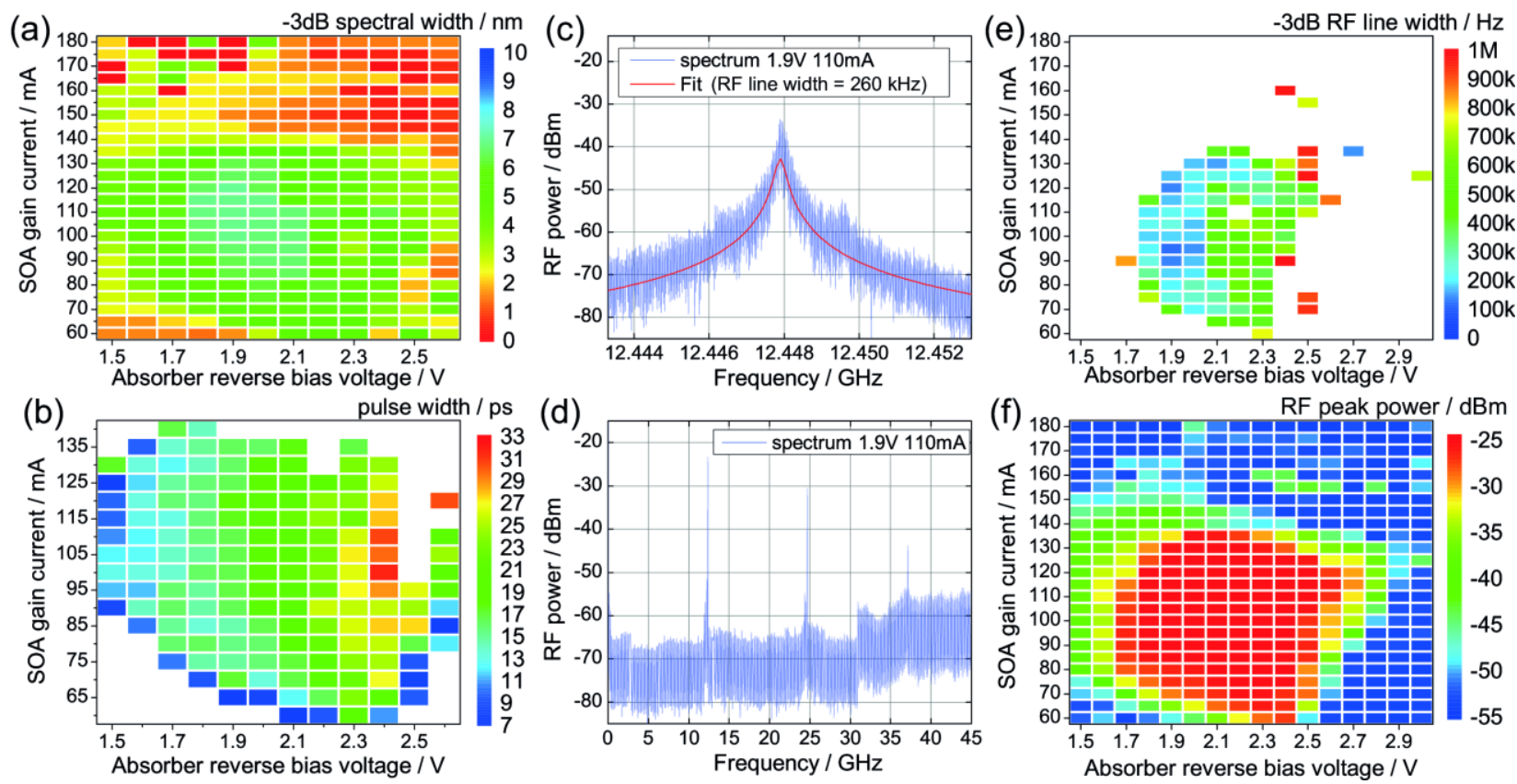

Fig. 2: (a) Map of -3-dB spectral bandwidth, and (b) Map of deconvoluted pulse width. (c) Zoom into fundamental RF beat tone, and (d) Wide-span RF spectrum for $\mathrm{I}_{\mathrm{SOA}}=110 \mathrm{~mA}, \mathrm{~V}_{\mathrm{SA}}=1.9 \mathrm{~V}$. (e) Map of -3-dB RF line width. (f) Map of RF beat tone power.

exceed $4 \mathrm{~nm}$. For the biasing regime of $\mathrm{I}_{\mathrm{SOA}}=80-120 \mathrm{~mA}$ and $\mathrm{V}_{\mathrm{SA}}=1.8-2.1 \mathrm{~V}$, the spectral bandwidth can exceed 6 nm. Fig. 2(b) presents the deconvoluted pulse width map where the shortest pulses $(7-8 \mathrm{ps})$ are found at the mode locking region boundary while the longest pulses (31 - $32 \mathrm{ps)}$ at $\mathrm{I}_{\mathrm{SOA}}=100 \mathrm{~mA}$ and applied voltage $\mathrm{V}_{\mathrm{SA}}=2.4 \mathrm{~V}$ ranging from 7 to 35 ps for the various operating injection current ISOA $_{S}$ and applied voltage $\mathrm{V}_{\mathrm{SA}}$.

For the aforementioned bias condition $\mathrm{I}_{\mathrm{SOA}}=110 \mathrm{~mA}$ and $\mathrm{V}_{\mathrm{SA}}=1.9 \mathrm{~V}$, we identify a narrow RF signal at $12.448 \mathrm{GHz}$ with a signal-to-noise ratio of $40 \mathrm{~dB}$ and an RF linewidth of $260 \mathrm{kHz}$ as revealed in Fig. 2(c). The full-span spectrum depicted in Fig. 2(d) clearly shows the harmonic tones. Similarly, the -3-dB RF line width and RF beat tone power are color-coded for ISOA varied from 60 to $180 \mathrm{~mA}$ in increments of $5 \mathrm{~mA}$ and $\mathrm{V}_{\mathrm{SA}}$ from 1.5 to $3.0 \mathrm{~V}$ in increments of $1.0 \mathrm{~V}$ in Fig. 2(e) and Fig. 2(f), respectively.

Within the range of $\mathrm{I}_{\mathrm{SOA}}=70-130 \mathrm{~mA}$ and $\mathrm{V}_{\mathrm{SA}}=1.8-$ $2.3 \mathrm{~V}$, the collected RF line width is below $600 \mathrm{kHz}$ and the RF power exceeds $-30 \mathrm{dBm}$. Within the range of $\mathrm{I}_{\mathrm{SOA}}$ from 70 to $130 \mathrm{~mA}$ and $\mathrm{V}_{\mathrm{SA}}$ from 1.8 to $2.1 \mathrm{~V}$, the collected $\mathrm{RF}$ linewidth is below $300 \mathrm{kHz}$.

\section{CONCLUSION}

We have developed a MLL array PIC in an InP generic integration technology and experimentally demonstrated the mode-locking behavior of a ring laser in the two-element array. 7-nm-wide optical frequency combs have been generated with a comb spacing of $12.448 \mathrm{GHz}$. The RF tone exhibits a line width of $260 \mathrm{kHz}$, and for the same bias condition we have achieved a pulse width of 14.5 ps. Given that the coherent comb bandwidth is sufficiently broad, further investigations for spectral tuning are of significant interest. Hybrid mode locking and controlling the intra-cavity EOPMs for both ring MLL will shortly be performed.

\section{ACKNOWLEDGMENT}

European Union's Horizon 2020 Marie Skłodowska-Curie grant agreement No. 642355 FiWiN5G. European Union's Horizon 2020 Marie Skłodowska-Curie grant agreement No. 713694 MULTIPLY. Spanish Ministerio de Economia y Competitividad through Programa Estatal de Investigación, Desarrollo e Inovación Orientada a los Retos de la Sociedad (grant iTWIT, TEC2016-76997-C3-3-R). DFG (German Research Foundation). Personal grant by Adolf Messer Foundation.

\section{REFERENCES}

[1] A. Dutt, C. Joshi, X. Ji, J. Cardenas, Y. Okawachi, K. Luke, A. L. Gaeta, and M. Lipson, "On-chip dualcomb source for spectroscopy," Science Advances 4 (2018).

[2] S. Latkowski, V. Moskalenko, S. Tahvili, L. Augustin, M. Smit, K. Williams, and E. Bente, "Monolithically integrated $2.5 \mathrm{GHz}$ extended cavity mode-locked ring laser with intracavity phase modulators," Opt. Lett. 40, 77-80 (2015).

[3] L. M. Augustin, R. Santos, E. den Haan, S. Kleijn, P. J. A. Thijs, S. Latkowski, D. Zhao, W. Yao, J. Bolk, H. Ambrosius, S. Mingaleev, A. Richter, A. Bakker, and T. Korthorst, "Inp-based generic foundry platform for photonic integrated circuits," IEEE Journal of Selected Topics in Quantum Electronics 24, 1-10 (2018). 